\title{
Lyapunov-type inequalities for certain higher order fractional differential equations
}

\author{
Xuhuan Wang ${ }^{\mathrm{a}, *}$, Youhua Peng ${ }^{\mathrm{b}}$, Wanchun $\mathrm{Lu}^{\mathrm{b}}$ \\ ${ }^{a}$ Department of Education Science, Pingxiang University, Pingxiang, Jiangxi 337055, China. \\ ${ }^{b}$ Department of Mathematics, Pingxiang University, Pingxiang, Jiangxi 337055, China.
}

Communicated by R. Saadati

\begin{abstract}
This paper generalizes the well-known Lyapunov-type inequality for certain higher order fractional differential equations. The investigation is based on a construction of Green's functions and finding its corresponding maximum value. As an application, we obtain a lower bound for the eigenvalues of corresponding equations. (c)2017 All rights reserved.
\end{abstract}

Keywords: Fractional differential equations, Lyapunov-type inequality, Green's function, boundary value problem. 2010 MSC: 34B37, 34B15, 34A08.

\section{Introduction}

In recent years, the investigation of the fractional order differential equations have attracted much attention. There have been many works on the solutions of nonlinear fractional order differential equations, and we refer the readers to the articles $[2,3,22,25]$. In addition, the study of Lyapunov inequalities for fractional equations has attracted much attention.

The well-known Lyapunov inequality [16] states that if $y(t)$ is a solution of

$$
y^{\prime \prime}(t)+q(t) y(t)=0
$$

with $y(a)=0=y(b)(a<b)$ and $y(t) \neq 0$ for $t \in(a, b)$, then

$$
\int_{a}^{b}|q(t)| d t>\frac{4}{b-a} .
$$

Lyapunov-type inequalities are very important. This type of inequality and many of its generalizations have proved to be useful tools in eigenvalue problems, oscillation theory and numerous other applications for the theories of differential and difference equations, such as to higher order differential equations,

\footnotetext{
*Corresponding author

Email addresses: wangxuhuan84@163.com (Xuhuan Wang), pengyouh@126.com (Youhua Peng), luwanchun540@163.com (Wanchun $\mathrm{Lu}$ )

doi:10.22436/jnsa.010.09.42
} 
delay differential equations, even-order differential equations, odd-order differential equations, discrete or continuous differential equations, linear Hamiltonian systems and so on (see Refs. [6, 10, 18-20, 24, 2629], and references therein).

In [7], Ferreira obtained that the following fractional differential equation,

$$
\left({ }_{a} D^{\alpha} y\right)(t)+q(t) y(t)=0, \quad a<t \leqslant b, 1<\alpha \leqslant 2, \quad y(a)=0=y(b),
$$

has a nontrivial solution, where $q$ is a real and continuous function, then

$$
\int_{a}^{b}|q(s)| d s>\Gamma(\alpha)\left(\frac{4}{b-a}\right)^{\alpha-1} \text {. }
$$

In [8], Ferreira obtained the Caputo's type fractional order differential equation has a nontrivial solution

$$
\int_{a}^{b}|q(s)| d s>\frac{\Gamma(\alpha) \alpha^{\alpha}}{[(\alpha-1)(b-a)]^{\alpha-1}} .
$$

In [17], O'Regan and Samet concerned with the problem of the Riemann-Liouville fractional order differential equation

$$
\left({ }_{a} D^{\alpha} u\right)(t)+q(t) u(t)=0, a<t<b, 3<\alpha \leqslant 4,
$$

together with the boundary conditions $u(a)=u^{\prime}(a)=u^{\prime \prime}(a)=0, u^{\prime \prime}(b)=0$, which has a nontrivial solution

$$
\int_{a}^{b}|q(s)| d s>\frac{\Gamma(\alpha)(\alpha-2)^{\alpha-2}}{2(\alpha-3)^{\alpha-3}(b-a)^{\alpha-1}}
$$

There are several extensions and generalizations of Lyapunov-type inequality. For some recent development on the topic, see $[1,4,5,9,11-15,21,23]$ and the references therein. However, there are few research results in Lyapunov-type inequality for the higher order fractional differential equation.

Motivated by the above works, we will consider the following fractional boundary value problems (FBVP's for short). More precisely, we will consider the following fractional differential equation

$$
\left\{\begin{array}{l}
{ }_{a} D^{\alpha} y(t)+q(t) y(t)=0, \quad a<t<b, \\
y(a)=y^{\prime}(a)=\cdots=y^{(n-2)}(a)=0, \quad y^{(n-2)}(b)=0,
\end{array}\right.
$$

where $2 \leqslant n-1<\alpha \leqslant n$, and $q:[a, b] \rightarrow R$ is a continuous function.

\section{Preliminaries}

In this section, we introduce preliminary facts and some basic results, which are used throughout this paper.

Definition 2.1 ([1]). The Riemann-Liouville fractional integral of order $\alpha$ is defined by

$$
\left(I_{a+}^{\alpha} f\right)(x)=\frac{1}{\Gamma(\alpha)} \int_{a}^{t}(t-s)^{\alpha-1} f(s) d s, \alpha>0, t \in[a, b],
$$

where $\Gamma$ is the gamma function.

Definition 2.2 ([1]). The Riemann-Liouville fractional derivative of order $\alpha$ is defined by

where $\mathrm{n}=[\alpha]+1$.

$$
\left(D_{a+}^{\alpha} f\right)(x)=\frac{1}{\Gamma(n-\alpha)}\left(\frac{d}{d t}\right)^{n} \int_{a}^{t}(t-s)^{n-\alpha-1} f(s) d s, t \in[a, b],
$$

Lemma 2.3 ([1]). Let $\alpha>0$. If $u \in C[a, b] \cap \mathrm{L}(\mathrm{a}, \mathrm{b})$, then the following equality holds

$$
I_{a+}^{\alpha} D_{a+}^{\alpha} u(t)=u(t)+\sum_{i=1}^{n} c_{i}(t-a)^{\alpha-i}
$$

for some constants $c_{i} \in R, i=1,2, \ldots, n$, where $n=[\alpha]+1$. 


\section{Main results}

We start by writing the following fractional order boundary value problem in its equivalent integral form.

Lemma 3.1. Let $\mathrm{y} \in \mathrm{C}[\mathrm{a}, \mathrm{b}], 2 \leqslant \mathrm{n}-1<\alpha<\mathrm{n}$, then the problem

$$
\left\{\begin{array}{l}
{ }_{a} D^{\alpha} y(t)+q(t) y(t)=0, \quad a<t<b \\
y(a)=y^{\prime}(a)=\cdots=y^{(n-2)}(a)=0, \quad y^{(n-2)}(b)=0,
\end{array}\right.
$$

has a unique solution

$$
y(t)=\int_{a}^{b} G(t, s) q(s) y(s) d s
$$

where

$$
G(t, s)=\frac{1}{\Gamma(\alpha)} \begin{cases}\frac{(t-a)^{\alpha-1}(b-s)^{\alpha-n+1}}{(b-a)^{\alpha-n+1}}-(t-s)^{\alpha-1}, & a \leqslant s \leqslant t \leqslant b, \\ \frac{(t-a)^{\alpha-1}(b-s)^{\alpha-n+1}}{(b-a)^{\alpha-n+1}}, & a \leqslant t \leqslant s \leqslant b .\end{cases}
$$

Proof. From Lemma 2.3, we obtain that

$$
y(t)=c_{1}(t-a)^{\alpha-1}+c_{2}(t-a)^{\alpha-2}+\cdots+c_{n}(t-a)^{\alpha-n}-\frac{1}{\Gamma(\alpha)} \int_{a}^{t}(t-s)^{\alpha-1} q(s) y(s) d s
$$

for some real constants $c_{i}, i=1,2, \ldots, n$. By the boundary conditions $y(a)=y^{\prime}(a)=\cdots=y^{(n-3)}(a)=0$, we get $c_{3}=c_{4}=\cdots=c_{n}=0$. By differentiating, we have

$$
\begin{aligned}
y^{(n-2)}(t)= & c_{1}(\alpha-1) \cdots(\alpha-n+2)(t-a)^{\alpha-n+1}+c_{2}(\alpha-1) \cdots(\alpha-n+1)(t-a)^{\alpha-n} \\
& -\frac{(\alpha-1) \cdots(\alpha-n+2)}{\Gamma(\alpha)} \int_{a}^{t}(b-s)^{\alpha-n+1} q(s) y(s) d s .
\end{aligned}
$$

The condition $y^{(n-2)}(a)=0$ yields $c_{2}=0$, and the condition $y^{(n-2)}(b)=0$ implies that

$$
c_{1}=\frac{1}{(b-a)^{\alpha-n+1} \Gamma(\alpha)} \int_{a}^{b}(b-s)^{\alpha-n+1} q(s) y(s) d s .
$$

Therefore, the unique solution of the fractional differential equation is

$$
y(t)=\frac{(t-a)^{\alpha-1}}{\Gamma(\alpha)(b-a)^{\alpha-n+1}} \int_{a}^{b}(b-s)^{\alpha-n+1} q(s) y(s) d s-\frac{1}{\Gamma(\alpha)} \int_{a}^{t}(t-s)^{\alpha-1} q(s) y(s) d s,
$$

which yields the desired result.

Lemma 3.2. The function $\mathrm{G}(\mathrm{t}, \mathrm{s})$ defined by (3.1) satisfies the following property:

$$
0 \leqslant G(t, s) \leqslant G(b, s)=\frac{(b-s)^{\alpha-n+1}(s-a)}{\Gamma(\alpha)} \sum_{i=1}^{n-2}(-1)^{i-1} C_{n-2}^{i}(b-a)^{n-2-i}(s-a)^{i-1},
$$

$(t, s) \in[a, b] \times[a, b]$, where $C_{n-2}^{i}$ is binomial coefficient.

Proof. By the definition of $\mathrm{G}$, let

and

$$
g_{1}(t, s)=\frac{(t-a)^{\alpha-1}(b-s)^{\alpha-n+1}}{(b-a)^{\alpha-n+1}}-(t-s)^{\alpha-1}, a \leqslant s \leqslant t \leqslant b
$$

$$
g_{2}(t, s)=\frac{(t-a)^{\alpha-1}(b-s)^{\alpha-n+1}}{(b-a)^{\alpha-n+1}}, a \leqslant t \leqslant s \leqslant b .
$$

We start with the function $g_{2}$. Compute the differentiating $g_{2}(t, s)$ with respect to $t$, we obtain 


$$
g_{2}^{\prime}(t, s)=\frac{(\alpha-1)(t-a)^{\alpha-2}(b-s)^{\alpha-n+1}}{(b-a)^{\alpha-n+1}} \geqslant 0
$$

Notably, $g_{2}(\cdot, s)$ is non-decreasing for all $t \in[a, b]$, hence $g_{2}$ satisfies the following inequalities

$$
g_{2}(b, s) \geqslant g_{2}(t, s) \geqslant 0, a \leqslant t \leqslant s \leqslant b .
$$

Now, we will verify that $g_{1}^{\prime}(t, s) \geqslant 0, a \leqslant s \leqslant t \leqslant b$.

$$
\begin{aligned}
g_{1}^{\prime}(t, s) & =\frac{(\alpha-1)(t-a)^{\alpha-2}(b-s)^{\alpha-n+1}}{(b-a)^{\alpha-n+1}-(\alpha-1)(t-s)^{\alpha-2}} \\
& =(\alpha-1)(t-a)^{\alpha-2}\left[\left(1-\frac{s-a}{b-a}\right)^{\alpha-n+1}-\left(1-\frac{s-a}{t-a}\right)^{\alpha-2}\right] \\
& \geqslant(\alpha-1)(t-a)^{\alpha-2}\left[\left(1-\frac{s-a}{b-a}\right)^{\alpha-n+1}-\left(1-\frac{s-a}{b-a}\right)^{\alpha-2}\right] \geqslant 0,
\end{aligned}
$$

which implies that $g_{1}(\cdot, s)$ is non-decreasing for all $t \in[a, b]$, hence we obtain that

$$
g_{1}(b, s) \geqslant g_{1}(t, s) \geqslant 0, a \leqslant s \leqslant t \leqslant b .
$$

Consequently, the function $G(t, s)$ is non-decreasing with respect to $t$, it follows that

$$
0 \leqslant G(t, s) \leqslant G(b, s)=\frac{(b-s)^{\alpha-n+1}(s-a)}{\Gamma(\alpha)} \sum_{i=1}^{n-2}(-1)^{i-1} C_{n-2}^{i}(b-a)^{n-2-i}(s-a)^{i-1} .
$$

Theorem 3.3. If there exists is a nontrivial continuous solution $\mathrm{y}(\mathrm{t})$ of the fractional boundary value problem

$$
\left\{\begin{array}{l}
{ }_{a} D^{\alpha} y(t)+q(t) y(t)=0, \quad a<t<b \\
y(a)=y^{\prime}(a)=\cdots=y^{n-2}(a)=0, \quad y^{(n-2)}(b)=0,
\end{array}\right.
$$

where $2 \leqslant n-1<\alpha \leqslant n, q$ is a real and continuous function, then

$$
\int_{a}^{b}(b-s)^{\alpha-n+1}(s-a) \sum_{i=1}^{n-2}(-1)^{i-1} C_{n-2}^{i}(b-a)^{n-2-i}(s-a)^{i-1}|q(s)| d s \geqslant \Gamma(\alpha) .
$$

Proof. Let $\mathrm{B}=\mathrm{C}[\mathrm{a}, \mathrm{b}]$ be the Banach space endowed with norm

$$
\|y\|_{\infty}=\max _{t \in[a, b]}|y(t)| .
$$

Using Lemma 2.3, we obtain that a nontrivial solution $y$ to the FBVP satisfies the integral equation

$$
y(t)=\int_{a}^{b} G(t, s) q(s) y(s) d s, t \in[a, b] .
$$

Obviously, $q$ cannot be the zero function on $[a, b]$ otherwise $y$ is a trivial solution. Thus, for all $t \in[a, b]$, we get

$$
|y(t)| \leqslant \int_{a}^{b}|G(t, s)\|q(s)\| y(s)| d s \leqslant\left(\int_{a}^{b} \sup _{a \leqslant t \leqslant b}|G(t, s) \| q(s)| d s\right)\|y\|_{\infty} .
$$


Since $y$ is nontrivial, then $\|y\|_{\infty} \neq 0$,

$$
1 \leqslant \int_{a}^{b} \sup _{a \leqslant t \leqslant b}|G(t, s) \| q(s)| d s .
$$

Now, an application of Lemma 3.2 yields

$$
1 \leqslant \int_{a}^{b}|G(b, s) \| q(s)| d s .
$$

Then we have

$$
\int_{a}^{b}(b-s)^{\alpha-n+1}(s-a) \sum_{i=1}^{n-2}(-1)^{i-1} C_{n-2}^{i}(b-a)^{n-2-i}(s-a)^{i-1}|q(s)| d s \geqslant \Gamma(\alpha),
$$

and we obtain the desired result.

Remark 3.4. Theorem 3.3 with $n=4$ reduces to Theorem 2.3 in [17].

Corollary 3.5. If the fractional boundary value problem (1.1) has a nontrivial continuous solution, then

$$
\int_{a}^{b}(b-s)^{\alpha-n+1}(s-a)|q(s)| d s \geqslant \frac{\Gamma(\alpha)}{(n-2)(b-a)^{n-3}} .
$$

Proof. For Theorem 3.3, we obtain the following integral equation

$$
\int_{a}^{b}(b-s)^{\alpha-n+1}(s-a) \sum_{i=1}^{n-2}(-1)^{i-1} C_{n-2}^{i}(b-a)^{n-2-i}(s-a)^{i-1}|q(s)| d s \geqslant \Gamma(\alpha),
$$

we note that

$$
\sum_{i=1}^{n-2}(-1)^{i-1} C_{n-2}^{i}(b-a)^{n-2-i}(s-a)^{i-1} \leqslant(n-2)(b-a)^{n-3} .
$$

Thus we have

$$
(n-2)(b-a)^{n-3} \int_{a}^{b}(b-s)^{\alpha-n+1}(s-a)|q(s)| d s \geqslant \Gamma(\alpha),
$$

which yields the desired result.

Remark 3.6. Corollary 3.5 with $\alpha=4$ reduces to Corollary 2.7 in [17].

Corollary 3.7. If the fractional boundary value problem (1.1) has a nontrivial continuous solution, then

$$
\int_{a}^{b}|q(s)| d s \geqslant \frac{\Gamma(\alpha)(\alpha-n+2)^{\alpha-n+2}}{(n-2)(\alpha-n+1)^{\alpha-n+1}(b-a)^{\alpha-1}} .
$$

Proof. Let

$$
\varphi(s)=(b-s)^{\alpha-n+1}(s-a), \quad s \in[a, b] .
$$

Now, differentiating $\varphi(s)$ on $(a, b)$, we obtain

$$
\varphi^{\prime}(s)=(b-s)^{\alpha-n}[(b-s)-(\alpha-n+1)(s-a)] .
$$

Notably, the function $\varphi$ has a maximum at point $s^{*}=\frac{b+(\alpha-n+1) a}{\alpha-n+2}$, that is

$$
\max _{a \leqslant s \leqslant b} \varphi(s)=\varphi\left(s^{*}\right)=(\alpha-n+1)^{\alpha-n+1}\left(\frac{b-a}{\alpha-n+2}\right)^{\alpha-n+2} .
$$


From Corollary 3.5, we have

$$
\int_{a}^{b} \varphi(s)|q(s)| d s \geqslant \frac{\Gamma(\alpha)}{(n-2)(b-a)^{n-3}}
$$

which yields

$$
\int_{a}^{b}|q(s)| d s \geqslant \frac{\Gamma(\alpha)}{(n-2)(b-a)^{n-3} \varphi\left(s^{*}\right)}
$$

which concludes the proof.

Remark 3.8. Corollary 3.7 with $\alpha=4$ reduces to Corollary 2.5 of [17].

Corollary 3.9. If there exists a nontrivial continuous solution to the fractional boundary value problem

$$
\left\{\begin{array}{l}
a D^{\alpha} y(t)+q(t) y(t)=0, a<t<b, 2<\alpha \leqslant 3 \\
y(a)=y^{\prime}(a)=y^{\prime}(b)=0
\end{array}\right.
$$

where $\mathrm{q}$ is a real and continuous function in $[\mathrm{a}, \mathrm{b}]$, then

$$
\int_{a}^{b}|q(s)| d s \geqslant \frac{\Gamma(\alpha)(\alpha-1)^{\alpha-1}}{(\alpha-2)^{\alpha-2}(b-a)^{\alpha-1}}
$$

The proof of the corollary is not difficult and hence is left to the reader as an exercise.

\section{Application}

In this section, we give an application of the above results for the eigenvalue problem. consider the problem

$$
\left\{\begin{array}{l}
\left({ }_{0} D^{\alpha} y\right)(t)+\lambda y(t)=0, \quad 0<t<1, \quad n-1<\alpha \leqslant n \\
y(a)=y^{\prime}(a)=\cdots=y^{(n-2)}(a)=0, \quad y^{(n-2)}(b)=0 .
\end{array}\right.
$$

Corollary 4.1. If $\lambda$ is an eigenvalue to the fractional boundary value problem (4.1), then

$$
|\lambda| \geqslant \frac{\Gamma(\alpha)(\alpha-n+3)(\alpha-n+2)}{(n-2)} .
$$

Proof. From Corollary 3.5 we have

$$
(n-2) \int_{0}^{1}(1-s)^{\alpha-n+1} s|\lambda| d s \geqslant \Gamma(\alpha),
$$

by the definition of Beta function, we get

$$
\int_{0}^{1}(1-s)^{\alpha-n+1} s d s=\int_{0}^{1}(1-s)^{(\alpha-n+2)-1} s^{2-1} d s=B(\alpha-n+2,2),
$$

where $B$ is the Beta function, and we obtain

$$
(n-2) B(\alpha-n+2,2)|\lambda| \geqslant \Gamma(\alpha) .
$$

Using the identity

$$
\mathrm{B}(x, y)=\frac{\Gamma(x) \Gamma(y)}{\Gamma(x+y)}
$$

we get

$$
|\lambda| \geqslant \frac{\Gamma(\alpha) \Gamma(\alpha-n+4)}{(n-2) \Gamma(\alpha-n+2) \Gamma(2)},
$$

which concludes the proof. 


\section{Acknowledgment}

The project is supported by NNSF of China (Grant No. 11661065) and Scientific Research Fund of Jiangxi Provincial Education Department (Grant No. GJJ151264).

\section{References}

[1] N. Al Arifi, I. Altun, M. Jleli, A. Lashin, B. Samet, Lyapunov-type inequalities for a fractional p-Laplacian equation, J. Inequal. Appl., 2016 (2016), 11 pages. 1, 2.1, 2.2, 2.3

[2] A. Ali, B. Samet, K. Shah, R. A. Khan, Existence and stability of solution to a toppled systems of differential equations of non-integer order, Bound. Value Probl., 2017 (2017), 13 Pages. 1

[3] A. Ali, K. Shah, R. A. Khan, Existence of positive solutions to a coupled system of nonlinear fractional order differential equations with m-point boundary conditions, Bull. Math. Anal. Appl., 8 (2016), 1-11. 1

[4] I. Cabrera, B. Lopez, K. Sadarangan, Lyapunov type inequalities for a fractional two-point boundary value problem, Math. Methods Appl. Sci., 40 (2017), 3409-3414. 1

[5] I. Cabrera, K. Sadarangani, B. Samet, Hartman-Wintner-type inequalities for a class of nonlocal fractional boundary value problems, Math. Methods Appl. Sci., 40 (2017), 129-136. 1

[6] D. Çakmak, Lyapunov-type integral inequalities for certain higher order differential equations, Appl. Math. Comput., 216 (2010), 368-373. 1

[7] R. A. C. Ferreira, A Lyapunov-type inequality for a fractional boundary value problem, Fract. Calc. Appl. Anal., 16 (2013), 978-984. 1

[8] R. A. C. Ferreira, On a Lyapunov-type inequality and the zeros of a certain Mittag-Leffler function, J. Math. Anal. Appl., 412 (2014), 1058-1063. 1

[9] R. A. C. Ferreira, Some discrete fractioal Lyapunov-type inequalities, Fract. Differ. Calc., 5 (2015), 87-92. 1

[10] K. Ghanbari, Y. Gholami, Lyapunov type inequalities for fractional Sturm-Liouville problems and fractional hamiltonian systems and applications, J. Fract. Calc. Appl., 7 (2016), 176-188. 1

[11] M. Jleli, R. Lakhdar, B. Samet, A Lyapunov-type inequality for a fractional differential equation under a Robin boundary condition, J. Funct. Spaces., 2015 (2015), 5 pages. 1

[12] M. Jleli, J. J. Nieto, B. Samet, Lyapunov-type inequalities for a higher order fractional differential equation with fractional integral boundary conditions, Electron. J. Qual. Theory Differ. Equ., 2017 (2017), 17 pages.

[13] M. Jleli, B. Samet, Lyapunov-type inequalities for a Fractional Differential Equation with mixed boundary conditions, Math. Inequal. Appl., 18 (2015), 443-451.

[14] M. Jleli, B. Samet, Lyapunov-type inequalities for fractional boundary-value problems, Electron. J. Differential Equations, 2015 (2015), 11 pages.

[15] A. A. Kilbas, H. M. Srivastava, J. J. Trujillo, Theory and Applications of Fractional Differential Equations, Elsevier, Amsterdam, (2006). 1

[16] A. M. Liapunov, Probleme général de la stabilité du mouvement, Ann. Fac. Sci. Toulouse Sci. Math. Sci. Phys., 9 (1907), 203-474. 1

[17] D. O'Regan, B. Samet, Lyapunov-type inequalities for a class of fractional differential equations, J. Inequal. Appl., 2015 (2015), 10 pages. $1,3.4,3.6,3.8$

[18] B. G. Pachpatte, On Lyapunov-type inequalities for certain higher order differential equations, J. Math. Anal. Appl., 195 (1995), 527-536. 1

[19] N. Parhi, S. Panigrahi, On Liapunov-Type Inequality for Third-Order Differential Equations, J. Math. Anal. Appl., 233 (1999), 445-460.

[20] N. Parhi, S. Panigrahi, Lyapunov-type inequality for higher order differential equations, Math. Slovaca, 52 (2002), 31-46. 1

[21] J. Rong, C. Z. Bai, Lyapunov-type inequality for a fractional differential equation with fractional boundary conditions, Adv. Difference Equ., 2015 (2015), 10 pages. 1

[22] K. Shah, H. Khalil, R. A. Khan, Upper and lower solutions to a coupled system of nonlinear fractional differential equations, Progr. Fract. Differ. Appl., 2 (2016), 31-39. 1

[23] S. Sitho, S. K. Ntouyas, W. Yukunthorn, J. Tariboon, Lyapunov's type inequalities for hybrid fractional differential equations, J. Inequal. Appl., 2016 (2016), 13 pages. 1

[24] A. Tiryaki, Recent developments of Lyapunov-type inequalities, Adv. Dyn. Syst. Appl., 5 (2010), 231-248. 1

[25] X.-H. Wang, L. Lu, J.-T. Liang, Multiple solutions of nonlinear fractional impulsive integro-differential equations with nonlinear boundary conditions, Math. Slovaca, 66 (2016), 1105-1114. 1

[26] X.-J. Yang, On Lyapunov-type inequality for certain higher-order differential equations, Appl. Math. Comput., 134 (2003), 307-317. 1

[27] X.-J. Yang, Y.-I. Kim, K. Lo, Lyapunov-type inequality for a class of odd-order differential equations, J. Comput. Appl. Math., 234 (2010), 2962-2968.

[28] X.-J. Yang, K. Lo, Lyapunov-type inequality for a class of even-order differential equations, Appl. Math. Comput., 215 (2010), 3884-3890. 
[29] Q.-M. Zhang, X. H. Tang, Lyapunov-type inequalities for even order difference equations, Appl. Math. Lett., 25 (2012), 1830-1834. 1 